\title{
Five years and counting!
}

\author{
BL Kelsall $^{1}$
}

W e begin this new year with high expectations, and I am taking this opportunity to update you, our readers, on the progress, challenges, and future of the journal. First, I will share our successes.

Because of the efforts of a committed editorial board, willing reviewers, and an effective managerial and publishing team, Mucosal Immunology has reached new heights in terms of both scope and stature. I am happy to report that the number of submissions to Mucosal Immunology has more than doubled since 2009 and has increased more than $45 \%$ in the past year alone. These submissions have come from more than 30 countries, with a decreasing proportion coming from North America, reflecting broadening worldwide exposure and acceptance. Furthermore, as measured by our 2011 Institute for Scientific Information impact factor (IF) of 6.963, the articles we have published are now cited on average more often than those in all but 12 of 139 immunology journals and all but 7 of those that publish original research. This continuing upward trend in our IF indicates that articles published in Mucosal Immunology are increasingly recognized by the scientific community. Finally, on a more practical level, we have continued to improve our review and publication process and are now returning most initial decisions within 30 days of submission and publishing accepted manuscripts online in less than 25 days.

So what are the changes and challenges ahead? Both Jo Viney and Paul Garside have decided to step down from their positions, solely for personal and professional reasons.
Their judgement and guidance have been invaluable to the journal's success, and their contributions deserve our immense gratitude. They will both stay on as Associate Editors. Taking their roles as Deputy Editors in 2013 will be William Agace from the University of Lund and Marsha Wills-Karp from Johns Hopkins University, both of whom will be open and honest stewards of the journal's future. Another change ahead is a consequence of our success in attracting an increasing number of exceptional manuscripts. Starting in 2013, the size of each issue will increase substantially to accommodate for this growth, as well as for manuscripts already published online. We will also be considering the possibility of moving to an online only format in 2014. Furthermore, as a necessity, the review process will become more stringent, with the goal of accepting fewer manuscripts-those of higher potential impact. Therefore, given this new reality, a primary challenge for Mucosal Immunology will be to effectively manage the growth in submissions in a fair and efficient manner.

On a final note, I would like to encourage all of you to help us continue to make the journal an exciting forum for discussing all areas of mucosal immunology. We welcome not only your valuable original research, but also your suggestions for reviews, commentaries, and new formats for content and dialogue. Let us hear from you. We need to know how you think we can make the journal more interesting, valuable, and enjoyable.

CC 2013 Society for Mucosal Immunology 